\title{
Decision Model of Quality Supervision Six Sigma Project Based on ANFIS and Evidence Theory
}

\author{
Yang Jianjun, Yao Jie \\ Dept. of Management Science, Naval Univ. of Engineering, Wuhan, 430033, China
}

\begin{abstract}
In order to effectively improve the implementation effect of quality supervision six sigma project, it is crucial to select an appropriate project. Through combination of six sigma project implementation experience with expert evaluation, an approach is proposed to solve the problem of experts' insufficient knowledge about candidate projects. While ANFIS is utilized to evaluate some indicators with experience available, some indicators without experience available can be evaluated based on evidence theory. In the end, the results are weighted and aggregated to obtain the comprehensive evaluation results. This approach can effectively address the problem of experts' insufficient knowledge about candidate six sigma projects at the initial stage of six sigma management. The effectiveness and feasibility of this approach is illustrated with the selection of quality supervision six sigma project for shipbuilding.
\end{abstract}

Keywords: quality supervision; six sigma project decision; ANFIS, evidence theory

\section{I . INTRODUCTION}

As a guiding theory and approach for quality supervision, six sigma management can effectively achieve more systematic, focused and accurate quality supervision. Project selection is the primary task of six sigma management, and a scientific, reasonable and feasible evaluation model is the prerequisite for its success. The correct selection of six sigma project depends on two guarantees: 1. correct evaluation indicator system for the selection of six sigma project; 2. scientific, reasonable and feasible evaluation model. Based on the evaluation indicator system for six sigma project selection, this research focuses on the improvement of enterprise production management effectiveness, which is much different from the improvement of quality supervision effectiveness. Therefore, it is necessary to establish an evaluation indicator system for the purpose of quality supervision improvement and in accordance with the requirements of six sigma project selection for quality supervision. With regard to the establishment of evaluation model, the experience in enterprise production management projects is obviously significant in the selection of six sigma project for quality supervision, and the evaluation method should be able to utilize this important information fully. In addition, there is not much experience in the application of six sigma in quality supervision, so some indicators must be evaluated based on the experts. An evaluation model must be able to effectively handle the problem of cognitive uncertainness in expert evaluation.

Reference [1] employed data envelopment analysis (DEA) to improve the indicator system in six sigma project selection. Reference [2] combined FEMA and AHP to decide six sigma project based on the risk assessment of project failure. Reference [3] made use of the advantages of DEMATEL to deal with the causal relations among the indicators for selection of six sigma project. Clearly, the existing approaches cannot be directly applied in the selection of quality supervision six sigma project. For this reason, this paper establishes a quality supervision six sigma project decision evaluation indicator system based on the features of quality supervision and builds a corresponding decision model through the comprehensive utilization of adaptive neuro-fuzzy inference system (ANFIS) and evidence theory. 


\section{II . Quality Supervision Six Sigma ProJect DECISION INDICATOR SYSTEM}

According to the requirements of quality supervision, the objective of quality supervision effective improvement is broken down into three criteria, and each criterion is further divided into related targets. There are causal relations among three criteria. Each indicator is a part of the causal relation chain and complies with the objective of quality supervision.

\section{A. Evaluation indicators of quality supervision effective criterion}

The improvement of quality supervision effectiveness is the main target of quality supervision six sigma improvement. Quality supervision effective indicators are the core of the whole evaluation indicator system. Quality supervision requires the strict control of production process to ensure that construction and quality comply with technical status and requirements of related national standards, and imposes the strict requirements for the lead time of product. Therefore, the design of evaluation indicators in the dimension of quality supervision effectiveness should mainly focus on production quality, production cycle and production cost.

\section{B. Evaluation indicators of internal process criterion}

A key and competitive internal process is required to maintain the improvement of quality supervision effectiveness. The internal process indicators focus on the key indicators that have the highest influence on the objective of quality supervision effectiveness. Based on business process evaluation indicators [4], the dimension of internal process takes into account the supervision personnel, supervision time and supervision cost, etc. needed to realize quality supervision six sigma improvement.

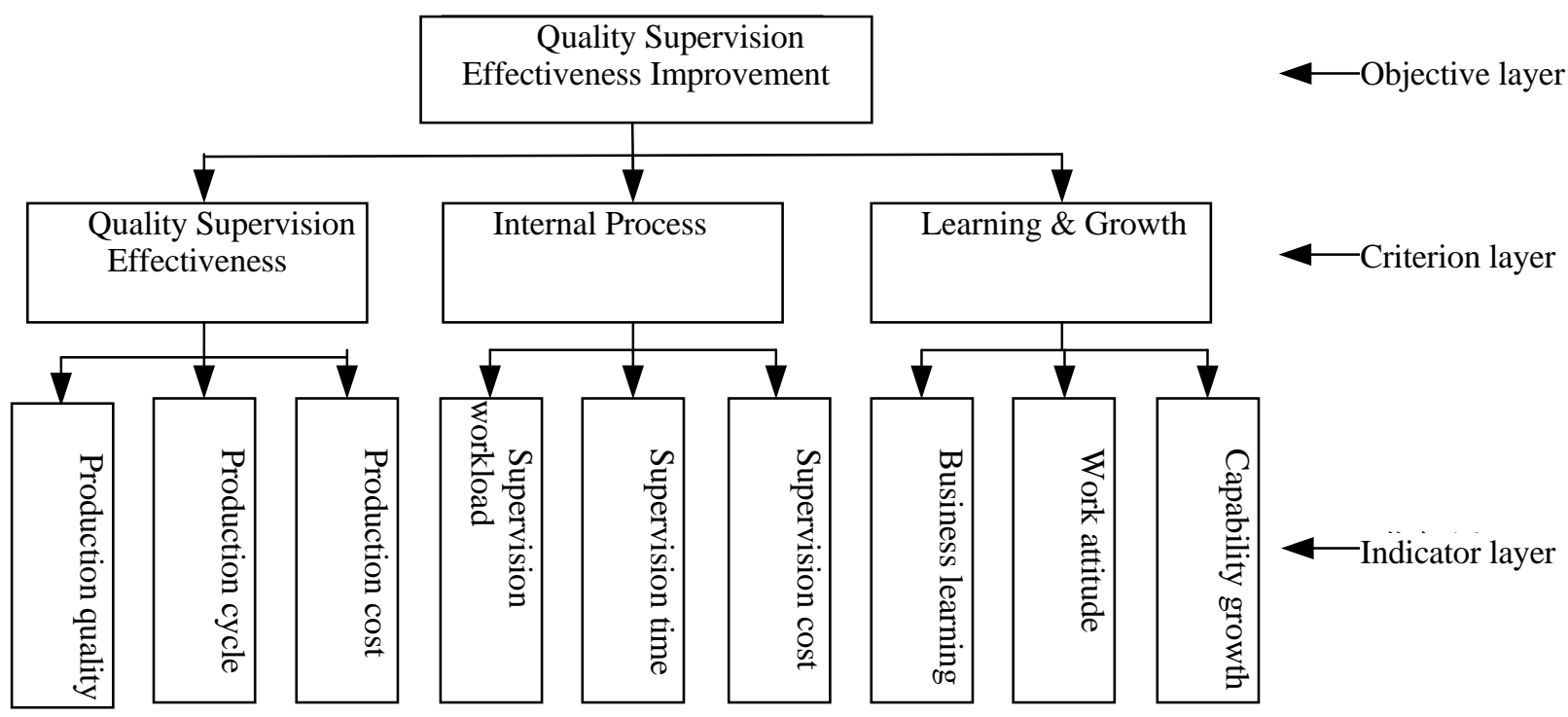

Fig. 1 Evaluation Indicator System for Selection of Quality Supervision Six Sigma Project

\section{Evaluation indicators of learning \& growth criterion}

Quality supervision effectiveness and internal process reveal the objective of quality supervision or the process capacity needed, but whether the objective can be realized and the gap between actual condition and expectation can be narrowed depends on an organization's learning \& growth capacity. Learning \& growth defines the foundation that an organization must make to drive its continuous growth. Based on the competence evaluation indicator system [5], learning \& growth criterion can be broken down into three indicators, namely, business learning, work attitude and capacity growth.

The evaluation indicator system for quality supervision six sigma project selection is presented in Fig. 1. 


\section{Quality Supervision Six Sigma Project Decision Model}

\section{A. Model Framework}

Based on the needs of modeling for quality supervision six sigma project decision, the quality supervision six sigma project is evaluated by combining the experience in the implementation of six sigma project with the expert evaluation in enterprise production. Some evaluation indicators with enterprise production experience available, the approach ANFIS is utilized for evaluation. First, empirical data are employed to train ANFIS model, and then the maturely trained model is utilized to obtain the evaluation results of such indicators. When some evaluation indicators have no experience for reference, evidence theory is utilized in the evaluation, and experts should give comments only on the indicators they have enough knowledge about. Then, the comments of experts are integrated to obtain the complete evaluation results of these indicators. Eventually, the evaluation results of such two approaches are weighted and aggregated to obtain the comprehensive evaluation of candidate project, and the six sig- ma project to be implemented firstly is selected based on the evaluation results.

Based on the evaluation indicator system of six sigma project, ANFIS approach is utilized to combine the evaluation information of quality supervision effectiveness. It is assumed that the evaluation result of every project is $y_{i}$. The information for evaluation of such indicators as internal process and learning \& growth is obtained based on the approach of evidence theory. It is assumed that the evaluation result of each project is $y_{i}^{\prime}$, the evaluation results of two indicators are weighted and aggregated, and the weights are given by expert panel. Assuming that the weights of $Y_{i}$ and $Y_{i}^{\prime}$ are $W_{i}$ and $w_{i}^{\prime}$ respectively and the comprehensive evaluation result of every project is $Y_{i}$, there is

$$
Y_{i}=w_{i} y_{i}+w^{\prime}{ }_{i}^{\prime}{ }_{i}
$$

The value of $Y_{i}$ determines the priority of each project.

\section{B. Quality Supervision Effectiveness Evaluation Model Based on ANFIS}

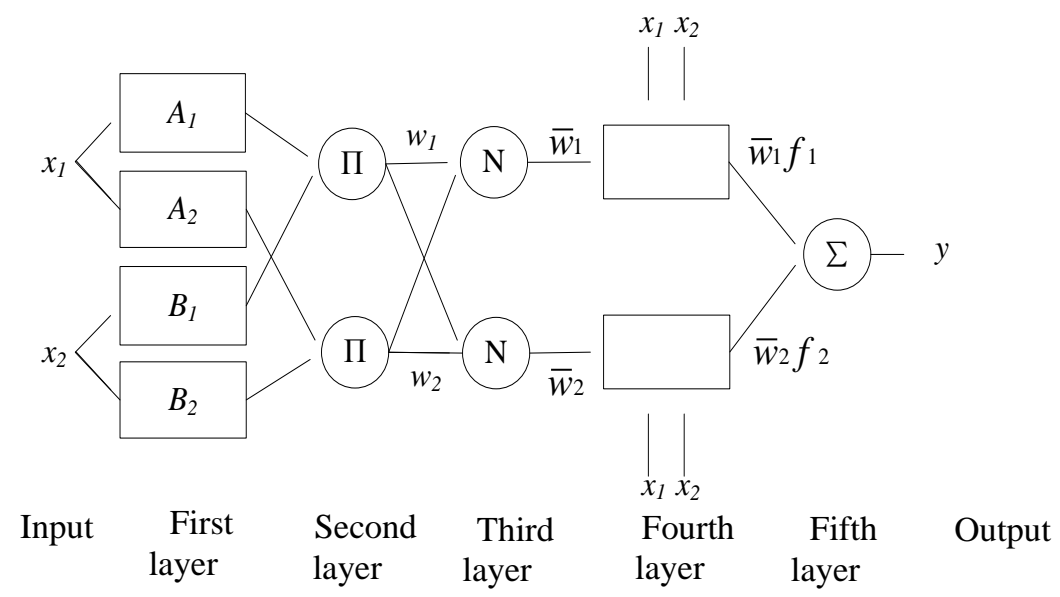

Fig. 2 Typical Structure of ANFIS

\section{1) Model Structure}

ANFIS is an organic combination of artificial neural network (ANN) and fuzzy inference system (FIS), so it offers the self-learning and adaptive capabilities of ANN and the reasoning capability of FIS. A typical structure of ANFIS is presented in Fig. 2 [6].

In the figure, each arrow indicates the flow direction of input signal, and each node indicates some functionality. There are square nodes and circular nodes. Square nodes contain adjustable parameters, which form a set of adjustable parameters in the adaptive neural network, while circular nodes do not contain adjustable parameters, but execute the specific functionalities. The empirical data from the implementation of six sigma project in the enterprise production are used to train the model, that is, adjust the values of adjustable parameters, so that the model can present the quality supervision effective evaluation closer to the actual condition. 
This paper builds the ANFIS model with T-S fuzzy reasoning. The outcome of comprehensive evaluation is the linear function for the value of quality supervision effective indicator in the evaluation. The 1-step T-S fuzzy reasoning rules are as follows:

$$
\text { if } x_{1} \text { is } A_{1} \text { and } x_{2} \text { is } B_{1} \text { then } y=p_{1} x_{1}+q_{1} x_{2}+r_{1}
$$

$$
\text { if } x_{1} \text { is } A_{2} \text { and } x_{2} \text { is } B_{2} \text { then } y=p_{2} x_{1}+q_{2} x_{2}+r_{1}
$$

The first layer is fuzzification layer, including adaptive nodes. Node parameter, that is, the parameter determining the shape of membership function, is called antecedent parameter. This layer is used to transform the value of every measuring indicator used by the enterprise to evaluate the quality supervision effectiveness into the membership function of every fuzzy set. Then, the fuzzification is completed.

$$
O_{i}^{1}=\mu_{A i}\left(x_{1}\right)
$$

$O_{i}^{\mathrm{j}}$ stands for the output of the ith node in the jth layer. $x_{1}$ is the output of the node $i$, that is, the value of measuring indicator for quality supervision effectiveness. $A_{i}$ and $B_{i}$ are fuzzy sets. The membership function may be any suitable parameterized membership function and its shape varies with the parameters. The parameters of such membership function are called antecedent parameters, so the variation of their values can change the shape of membership function. $\mu_{A i}$ is the value of $A_{i}$ in the membership function, representing the degree that $X$ is a member of $A_{i}$.

The second layer is the strength release layer of fuzzy rule, which represents the influence of every fuzzy rule on the comprehensive evaluation result of quality supervision effectiveness. This layer only executes the specific node functionalities and have no adjustable parameters. The $\Pi$ of circular nodes in the second layer means that the signals fuzzified in the first layer are intersected and then outputted. $w_{i}^{\prime}$ indicates the strength of fuzzy rule at the ith node.

$$
O_{i}^{2}=w_{i}^{\prime}=\mu_{A i}\left(x_{1}\right) \cdot \mu_{B_{j}}\left(x_{2}\right)
$$

The third layer is the normalization of rule strength,that is, calculating the ratio of the strength of fuzzy rule to the aggregate strength of all rules at the ith node. It represents the weight of influence that the output subject to this rule has on the comprehensive evaluation results of quality supervision effectiveness. The calculation formula is as follows:

$$
O_{i}^{3}=\bar{w}_{i}^{\prime}=\frac{w_{i}^{\prime}}{w_{1}^{\prime}+w_{2}^{\prime}} \mathrm{i}=1,2
$$

The fourth layer is the output of fuzzy rule, which represents the result obtained after inputting the values of quality supervision effective indicators according to a fuzzy rule. Each node is adaptive. Node parameter $\left\{p_{i}, q_{i}, r_{i}\right\}$ is called consequent parameter. The output formula is as follows:

$$
O_{i}^{4}=\bar{w}_{i}^{\prime} f_{i}=\bar{w}_{i}^{\prime}\left(p_{i} X_{1}+q_{i} X_{2}+r_{i}\right) i=1,2
$$

The fifth layer is to calculate the total output of all input signals, i.e. the comprehensive effectiveness of project based on the values of various indicators. The calculation formula is as follows:

$$
O_{i}^{5}=\sum_{i} \bar{w}_{i}^{\prime} f_{i}=\frac{\sum_{i} w_{i}^{\prime} f_{i}}{\sum_{i} w_{i}}
$$

ANFIS is a typical adaptive network. While determining antecedent parameters, the total output of all signal inputs can be represented by the linear combination of consequent parameters, that is:

$$
\begin{aligned}
& O_{i}^{5}=\bar{w}_{1} f_{1}+\bar{w}_{2} f_{2} \\
& =\left(\bar{w}_{1} X_{1}\right) p_{1}+\left(\bar{w}_{1} X_{2}\right) q_{1}+\bar{w}_{1} r_{1} \\
& +\left(\bar{w}_{2} X_{1}\right) p_{2}+\left(\bar{w}_{2} X_{1}\right) q_{2}+\bar{w}_{2} r_{2}
\end{aligned}
$$

\section{2) Learning Pattern of the Model}

The initial structure for quality supervision effectiveness evaluation model based on ANFIS is established, which requires the determination of antecedent parameters and consequent parameters through learning. The model can describe the relationship of mapping between the values of quality supervision effectiveness measuring indicators and the evaluation results more accurately.

ANFIS learning algorithm is a hybrid algorithm. BP approach is utilized to adjust antecedent parameters while least squares estimate (LSE) is employed to adjust consequent parameters. The specific process is that learning is carried out forward to the fourth layer of ANFIS and then LSE approach is utilized to obtain consequent parameters. Signals are passed along the network forward to the fifth layer (output layer) to obtain output errors. BP approach is utilized for backward learning to fix the parameters in the output layer. The parameters in the intermediate layer or input layer are self-optimized in the direction of reducing output errors, and the antecedent parameters are altered based on the changes of returned errors, till the mean square error of sample data set reaches the specified requirements for accuracy ${ }^{[7]}$. 
3) Model Application Steps

ANFIS is applied in quality supervision effectiveness evaluation in the following steps:

Step 1: Generate training data pairs and testing data pairs. Sort out the historic data for the implementation of six sigma management in enterprise production, and establish the data pairs of values of quality supervision effective indicators and evaluation results. Use some data as testing data pairs, and the other data as training data pairs.

Step 2: Use genfis function to generate the initial FIS structural model. The number of input variables is the number of quality supervision effectiveness indicators, but there is only an output variable. Before the initial FIS is generated, the related parameters can use default values. Under the interface of Anfis edit, select the Sub.clustering (subtract clustering) in the edit area Generate FI.

Step 3: Use anfis function to train ANFIS. Use training data pairs to generate the initial FIS for training to modify and adjust the values of antecedent parameters and consequent parameters. The results of model fitting can better match with actual condition. Set Optim.Method to hybrid; set Error Tolerance to 0; and set Epochs to 50 (maximum training times).

Step 4: Use testing data to verify the performance of ANFIS. If the results satisfy the requirements for error, it is believed that the ANFIS system can well realize the mapping between the values of quality supervision effectiveness measuring indicators and evaluation results.

Step 5: Input the values of quality supervision effectiveness measuring indicators of candidate project into the trained ANFIS to obtain the comprehensive evaluation results of quality supervision effectiveness of the project.

\section{Internal Process and Learning \& Growth Evaluation Model Based on Evidence Theory}

As there are not historic data for two indicators, i.e. internal process and learning \& growth, evidence theory is introduced to solve the problem that experts have no sufficient knowledge about candidate six sigma projects. Experts are only required to evaluate the indicators they have sufficient knowledge about, and then combine the comments of experts, so as to obtain the comprehensive and highly trustworthy evaluation results for the same problem.

The set of candidate six sigma projects is represented by identification framework $\Theta$ in evidence theory. All elements in $\Theta$ are exclusive to each other. It is presented as follows:

$$
\Theta=\left\{\theta_{1}, \theta_{2}, \cdots \quad \theta_{n}\right\}
$$

Let $A$ be any subset of the identification framework, there is $A \subseteq \Theta$ and it satisfies:

$$
\left\{\begin{array}{l}
m(\phi)=0 \\
\sum_{A \subset \Theta} m(A)=1
\end{array}\right.
$$

In which, $m(A)$ is the basic belief assignment function (BPA) of the event $A$, indicating the degree that the evidence has belief in $A$, that is, believability. The believability of empty set is 0 . Experts have uncertain knowledge about candidate projects, so they are required to evaluate each project separately, in order to guarantee the reliability of evaluation information. Therefore, $A$ stands for a single element set, that is, candidate project.

For multiple belief assignment functions $m_{1}, m_{2}, \cdots, m_{n}$, orthogonal calculation can be conducted to obtain a belief assignment function. If $m_{1}, m_{2}, \cdots, m_{n}$ are $\mathrm{n}$ basic belief functions on the same identification framework $\Theta$, the corresponding independent evidences are $E_{1}, E_{2}, \cdots, E_{n}$ and the focus is $A_{i}^{j}(i=1,2, \cdots, n ; j=1,2, \cdots)$. Thus, the evidence combination rule [8] is as follows:

$$
\begin{cases}\frac{\sum_{\cap A_{i}=A} \prod m_{i}\left(A_{i}^{j}\right)}{1-K} & A \neq \varnothing \\ 0 & A=\varnothing\end{cases}
$$

In which,

$$
K=\sum_{\cap A_{i}=\Phi} \prod m_{i}\left(A_{i}^{j}\right)
$$

As shown in Equations (1) (3), the combination rule of evidence theory is certain, and the evaluation results are determined by the believability of evidences. In other words, the reliability of values assigned to believability directly decides the reliability of evaluation results. The evaluation model is built in two steps:

Step 1: Assign values to believability of evidence

In the evidence theory, evidence is not physical, but a portion of knowledge and experience and the 
results from the observation and research of a specific problem. An expert analyzes the evidence to conclude own opinion on the problem. The evidence theory can solve the problem of uncertain knowledge since it does not request experts to have complete knowledge about candidate six sigma projects. Each expert may, based on the field he is familiar with, evaluate a candidate project using the fully recognized indicators. Then, the comments of all experts are combined. The comment of experts on every candidate project represents the believability of their recognition regarding the priority of the projects.

\section{Step 2: Combine evidences}

The value of believability assigned by experts to each candidate project is submitted into Equation (11) and Equation (12) to obtain the comprehensive evaluation results of expert panel with regard to the candidate project.

\section{IV.Case Analysis}

In the quality supervision of shipbuilding, quality supervisor notifies production department of any problem discovered during patrol inspection by means of Notice of Quality Supervision Comments, requiring its rectification. The problems on the Notices of Quality Supervision Comments within a given period are summed up to classify the quality problems requiring particular rectification into five categories as follows: drawing problems ( $\theta_{1}$ ), painting problems $\left(\theta_{2}\right)$, welding problems $\left(\theta_{3}\right)$, accuracy problems $\left(\theta_{4}\right)$ and piping problems $\left(\theta_{5}\right)$. The five categories of problems are taken as quality supervision six sigma projects. $g_{1} \sim g_{16}$ represent the indicators in the indicator system respectively. $B_{1}, B_{2}, B_{3}$ and $B_{4}$ represent the experts participating in the selection of shipbuilding quality supervision six sigma project.

\section{A. Shipbuilding Quality Supervision Effectiveness Evaluation}

The empirical data from the enterprise's implementation of six sigma project in production are sorted out to gain the data pairs in Table 1 . The first 15 data pairs are taken as the training data pairs, while the last data pair is used as the testing data pair. Expert panel gives comments on the quality supervision effectiveness indicators of candidate projects as presented in Table 2.

TABLE 1 EMPIRICAL DATA OF QUALITY SUPERVISION EFFECTIVENESS

\begin{tabular}{|c|c|c|c|c|c|c|c|c|c|c|c|c|c|c|c|c|}
\hline$\Theta$ & 1 & 2 & 3 & 4 & 5 & 6 & 7 & 8 & 9 & 10 & 11 & 12 & 13 & 14 & 15 & 16 \\
\hline$g_{1}$ & 3 & 3 & 4 & 4 & 4 & 3 & 3 & 4 & 5 & 5 & 5 & 5 & 6 & 6 & 6 & 6 \\
\hline$g_{2}$ & 3 & 4 & 4 & 3 & 4 & 4 & 4 & 5 & 4 & 5 & 6 & 5 & 6 & 6 & 5 & 4 \\
\hline$g_{3}$ & 3 & 3 & 3 & 3 & 4 & 4 & 5 & 4 & 4 & 5 & 5 & 6 & 5 & 6 & 5 & 5 \\
\hline Result & 65 & 68 & 73 & 70 & 75 & 70 & 75 & 80 & 83 & 88 & 93 & 90 & 95 & 98 & 93 & 90 \\
\hline
\end{tabular}

TABle 2 COMMENTS OF EXPERT PANEL ON QUALITY SUPERVISION EFFECTIVENESS INDICATORS OF CANDIDATE PROJECTS

\begin{tabular}{llllll}
\hline & $\theta_{1}$ & $\theta_{2}$ & $\theta_{3}$ & $\theta_{4}$ & $\theta_{5}$ \\
\hline$g_{1}$ & 6 & 3 & 4 & 4 & 3 \\
\hline$g_{2}$ & 5 & 4 & 5 & 6 & 4 \\
\hline$g_{3}$ & 4 & 5 & 4 & 4 & 3 \\
\hline
\end{tabular}


After the training is completed, the interface shows "Epoch50: error=5.2179e-006", and the error satisfies the requirements. Empirical data are inputted into the trained ANFIS to obtain the result 88.8, which is very close to the original result 88 . It means that the accuracy of this ANFIS satisfies the requirements. Based on Table 2, the results are obtained as presented in Table 3.

TABLE 3 EVALUation Results of CANDIDATE PROJECTS by EXPERTS

\begin{tabular}{llllll}
\hline$\Theta$ & $\theta_{1}$ & $\theta_{2}$ & $\theta_{3}$ & $\theta_{4}$ & $\theta_{5}$ \\
\hline$g^{1}$ & 6 & 3 & 4 & 4 & 3 \\
\hline$g_{2}$ & 5 & 4 & 5 & 6 & 4 \\
\hline$g_{3}$ & 4 & 5 & 4 & 4 & 3 \\
\hline Result & 91.6 & 75 & 80 & 86.7 & 68 \\
\hline Normalized & 0.2283 & 0.1869 & 0.1994 & 0.2160 & 0.1694 \\
\hline
\end{tabular}

B. Evaluation of Internal Process and Learning \& Growth for Shipbuilding

Using the indicators of internal process and learning \& growth $\left(g_{5} \sim g_{16}\right)$, experts assign the values of believability to candidate projects based on their own knowledge and experience as presented in Table 4. The results of evidence combination are obtained as presented in Table 5.

TABle 4 VAlues of Believability EXPERTS Assign to EVIDENCE OF CANDIDATE PROJECTS

\begin{tabular}{lccccc}
\hline$\Theta$ & $\theta_{1}$ & $\theta_{2}$ & $\theta_{3}$ & $\theta_{4}$ & $\theta_{5}$ \\
\hline$B_{1}$ & 0.21 & 0.21 & 0.19 & 0.16 & 0.23 \\
\hline$B_{2}$ & 0.25 & 0.24 & 0.15 & 0.17 & 0.19 \\
\hline$B_{3}$ & 0.22 & 0.22 & 0.19 & 0.19 & 0.18 \\
\hline$B_{4}$ & 0.21 & 0.24 & 0.17 & 0.18 & 0.20 \\
\hline
\end{tabular}

TABLE 5 RESUlTS OF EVIDENCE COMBINATION

\begin{tabular}{cccccc}
\hline Project & $\theta_{1}$ & $\theta_{2}$ & $\theta_{3}$ & $\theta_{4}$ & $\theta_{5}$ \\
\hline & 0.1081 & 0.3127 & 0.2851 & 0.1092 & 0.1849 \\
\hline
\end{tabular}

C. Comprehensive Evaluation Results of Shipbuilding Quality Supervision Six Sigma Project

The quality supervision effectiveness, internal process and learning \& growth of shipbuilding quality supervision six sigma project are evaluated. Based on the weights assigned by expert panel to these results, the comprehensive evaluation results are presented in Table 6. 
TABLE 6 COMPREHENSIVE EVALUATION RESUltS

\begin{tabular}{ccccccc}
\hline Project & $\theta_{1}$ & $\theta_{2}$ & $\theta_{3}$ & $\theta_{4}$ & $\theta_{5}$ & Weight \\
\hline ANFIS & 0.2283 & 0.1869 & 0.1994 & 0.2160 & 0.1694 & 0.65 \\
\hline Evidence Theory & 0.2851 & 0.3127 & 0.1081 & 0.1092 & 0.1849 & 0.35 \\
\hline Weighted Averaging & 0.2482 & 0.2309 & 0.1674 & 0.1786 & 0.1748 & \\
\hline
\end{tabular}

As presented in the above table, priority should be given to implementing the drawing six sigma improvement project. This matches with the significance of drawings in shipbuilding and the severe consequences of drawing errors. Drawings exist in the whole life of ship from building to decommissioning, so their correctness decides 50\% quality reliability of ship. If production department fails to modify the drawings of construction process in a timely manner with regard to the quality problem discovered by quality supervisor or other parties' request for or notice of modification to drawings, the quality problem may recur and cause very considerable losses of reworking.

\section{Conclusion}

This paper addresses the uncertain knowledge of experts about candidate six sigma projects and utilizes the ANFIS approach to comprehensively evaluate the quality supervision effectiveness indicators with the experience of enterprise production available, and the evidence theory to comprehensively evaluate the internal process and learning \& growth indicators without experience available. Then, the evaluation results are weighted and combined to obtain the comprehensive evaluation results. The approach proposed in this paper can effectively solve the problem of lacking historic data for some evaluation indicators in the selection of quality supervision six sigma project. Therefore, it can be also applied in the comprehensive evaluation when some indicators have training sample while some indicators depend only on expert evaluation.

\section{REFERENCES:}

[1] Kumar U D, Saranaa H, Ramirez J E. Six sigma project selection using data envelopment analysis[J]. The TQM Magazine, 2007, 9(5): 419-441.
[2] Su C T, Chou C. A systematic methodology for the creation of Six Sigma projects: A case study of semiconductor foundry[J]. Expert Systems with Applications, 2008(34): 2693-2703.

[3] Gülin Büyükozkan, Demet Oztürkcan. An integrated analytic approach for Six Sigma project selection[J]. Expert Systems with Applications, 2010(37): 5835-5847.

[4] Zeng Yimin. Design of Process-Oriented Key Performance Indicators System in the Power Supply Enterprise[D]. Shijiazhuang: North China Electric Power University, 2012.

[5] Pan Xiaoling. Establishment and valuation of human resource management model based on competency[J]. Enterprise Economy, 2011(8): 72-75.

[6] Wu Xingxing, Liu Jinguo. Improvement of Fuzzy System and ANFIS and Its Application in Space Optics[M]. Beijing: Science Press, 2012.

[7] Chen Xinbing. The Simulation of ANFIS by MATLAB and the Study of Hydraulic Pressure Machine Fuzzy Temperature Control System[D]. Changsha: Hunan University, 2005.

[8] Yang Fengbao, Wang Xiaoxia. Combination Method of Conflictive Evidences in D-S Evidence Theory[M]. Beijing: National Defense Industry Press, 2010. 